\title{
A BACl study of river restoration with large- wood additions using Pl tagged tracer particles to access bed-sediment mobility on the Narraguagus River, Maine
}

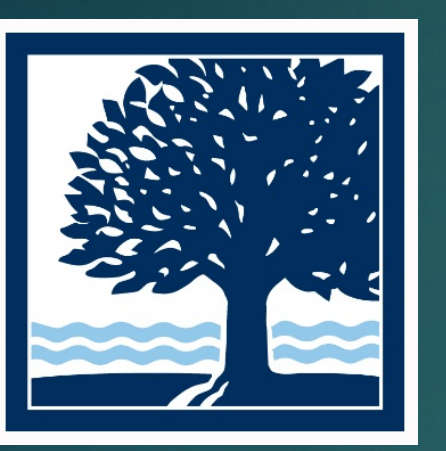

DOUGLAS THOMPSON' ${ }^{1}$, STEVEN KOENIG², SAMUEL FIXLER', ANNA MARSHALL ${ }^{3}$, MORIAH MCKENNA', EMMA BROOKS', JOSHUA COLEMAN' ${ }^{\prime}$ CLARE LOUGHLIN' \& CHARLIE WILLIAMS ${ }^{1}$

1 Environmental Studies Program, Connecticut College, New London, CT

2 Project SHARE, Eastport, ME

3 Connecticut Fund for the Environment, Save the Sound, New Haven, CT

Funding provided by the National Fish and Wildlife Foundation

(Grant \# 2004-0010-022 and 49552). Elizabeth Follett and Kalinda Roberts assisted with fieldwork. Chris Federico did much of the restoration design and implementation work. 
Narraguagus River, Maine USA: Atlantic salmon restoration

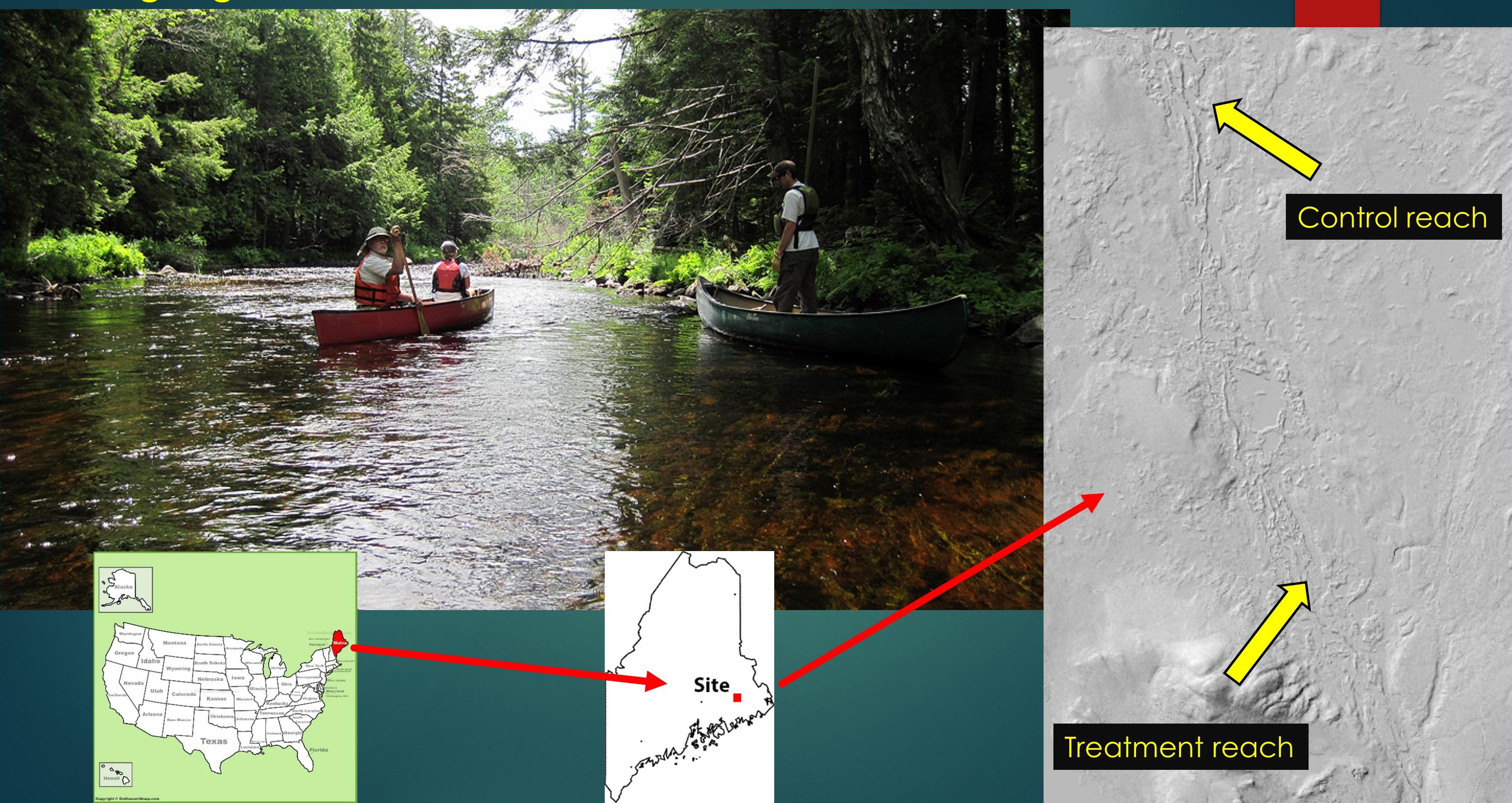




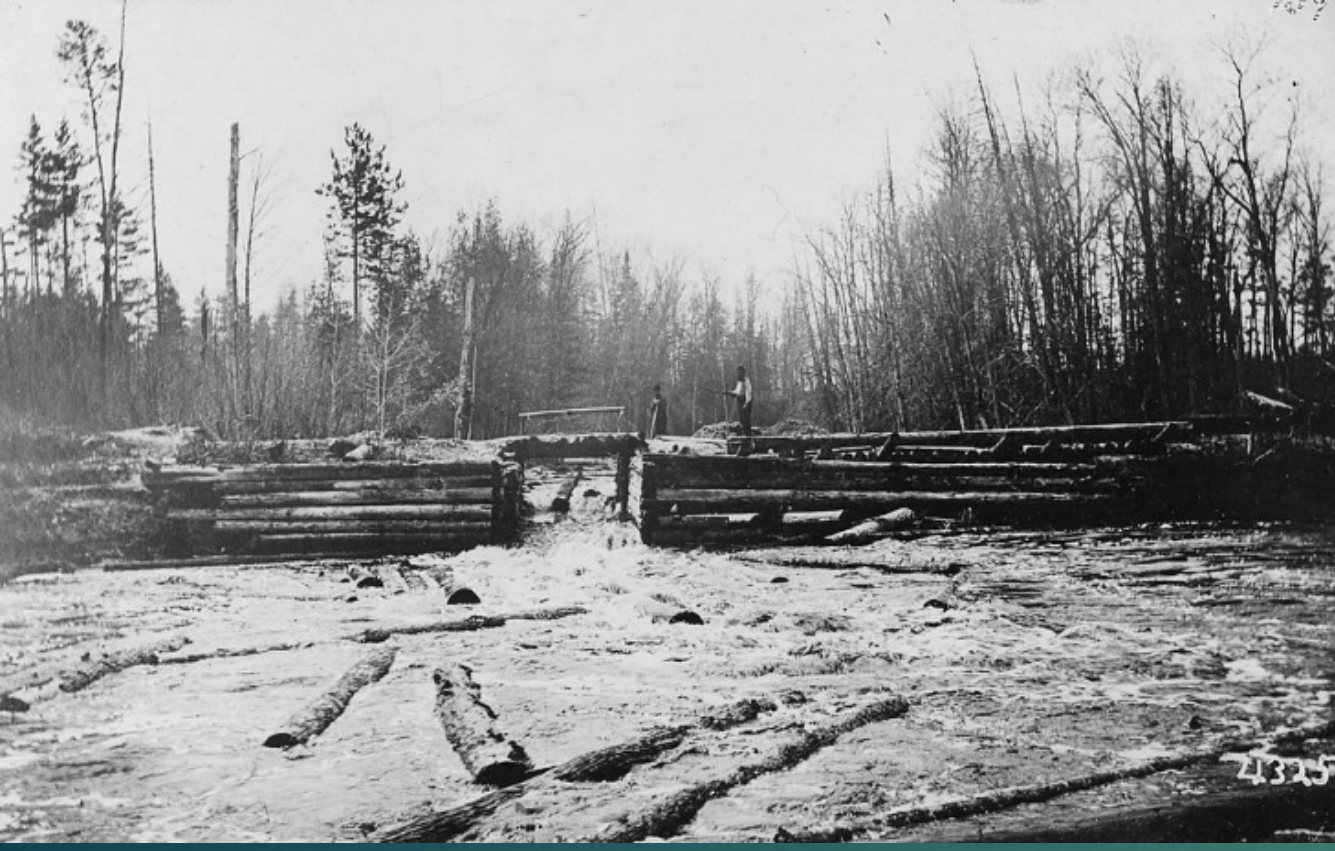

Photo courtesy Boston Archives

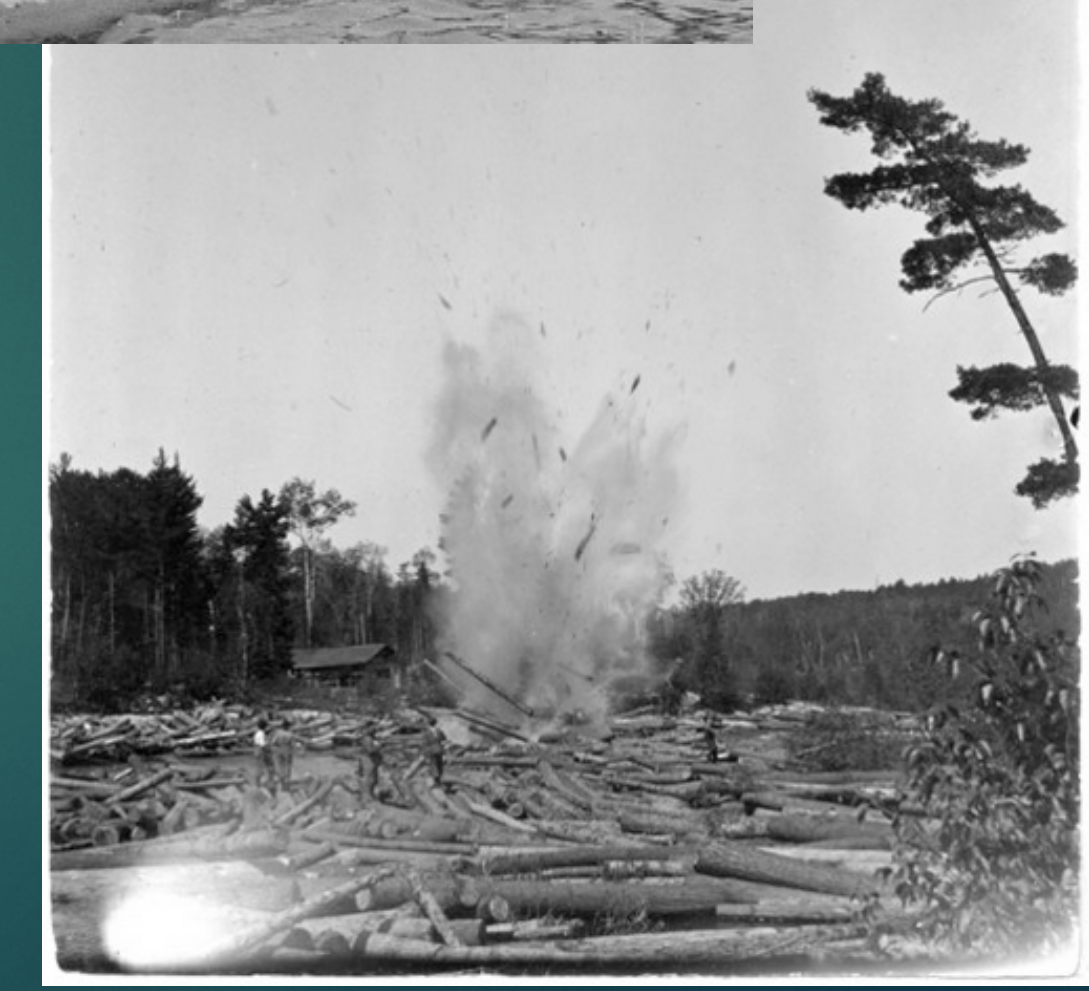

Photo courtesy Minnesota Historical Society collection, locator number HD5.44 r9. 


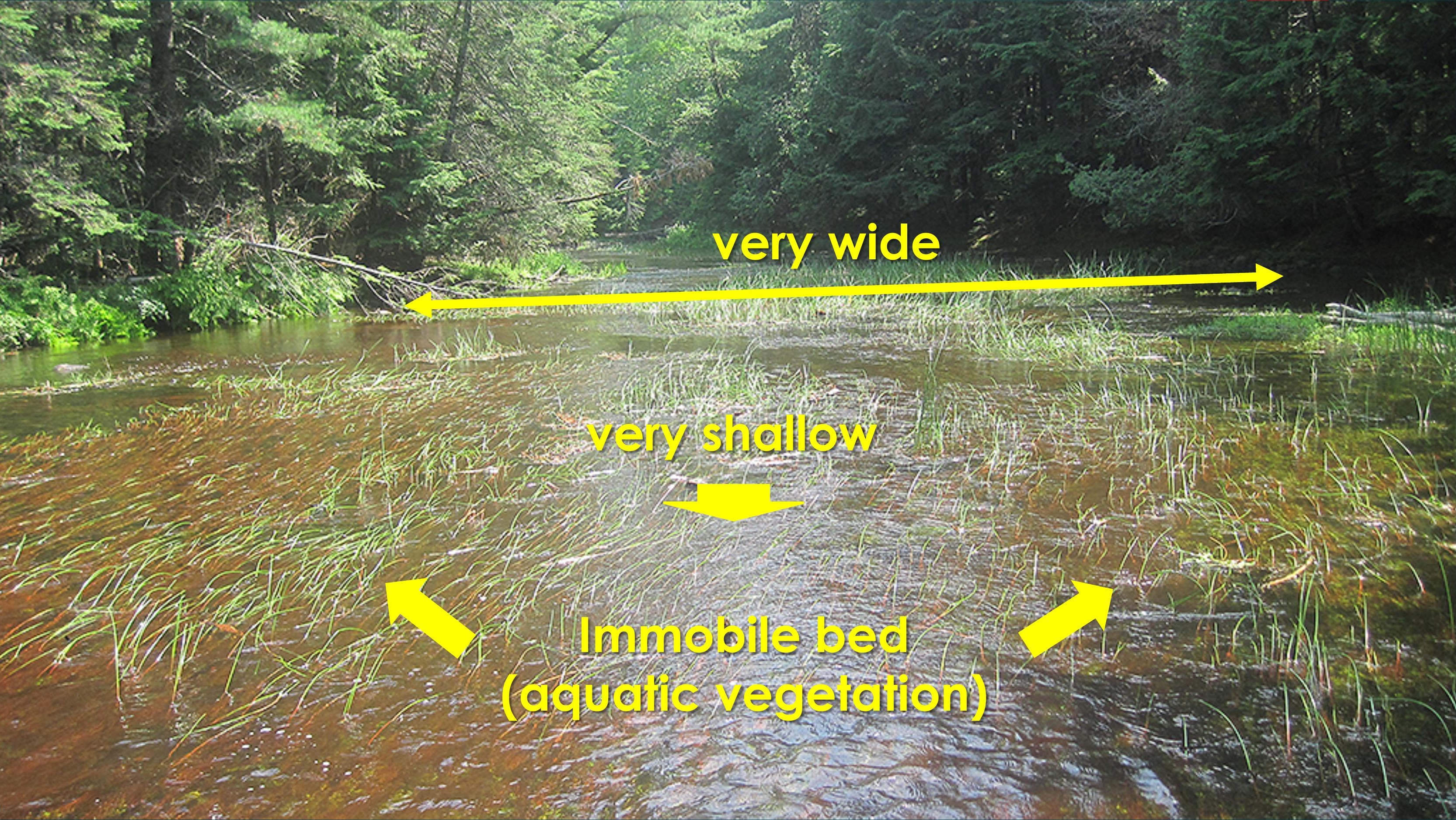




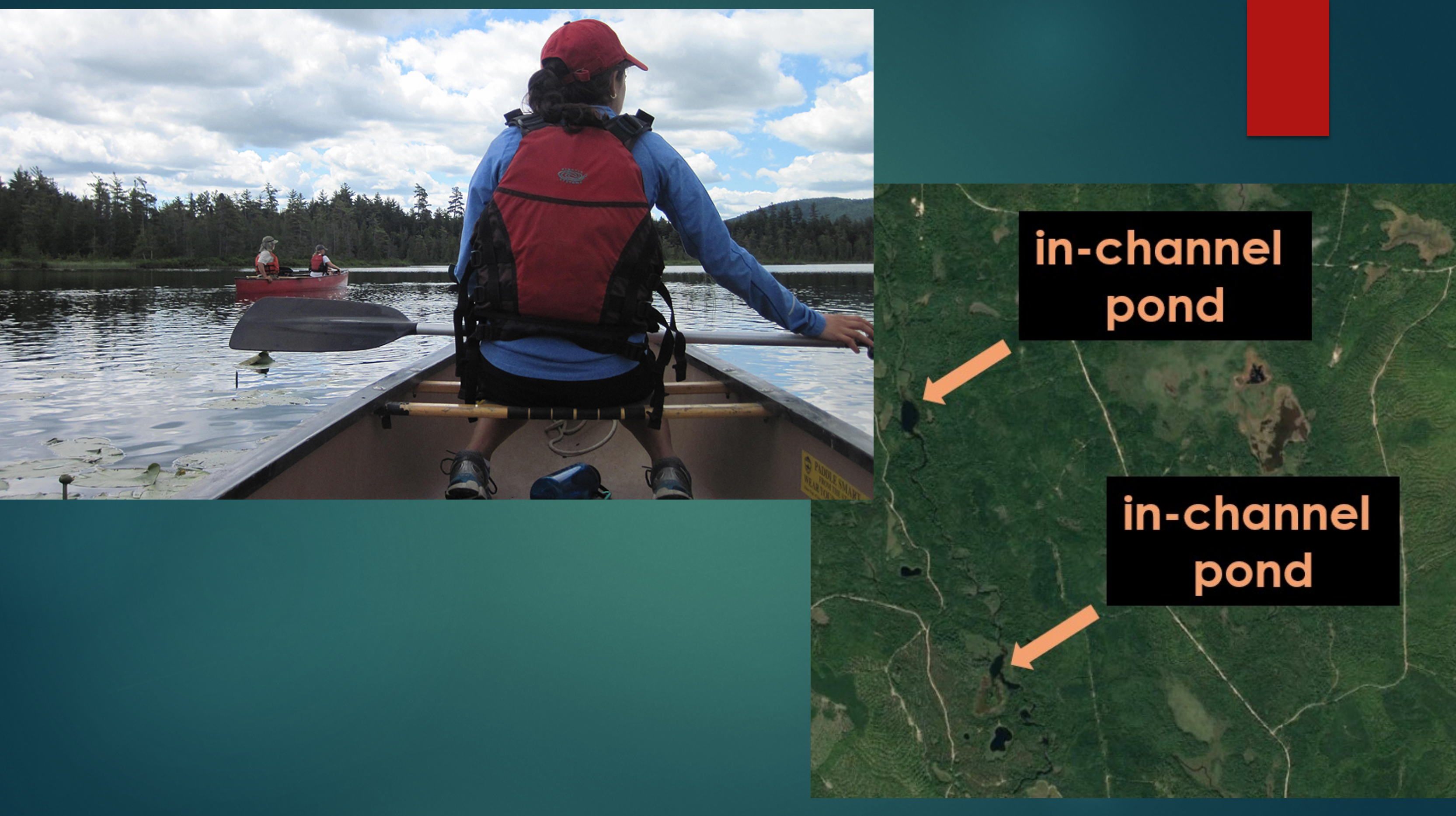




\section{How can wood additions help?}




\section{Narraguagus River, Maine USA}

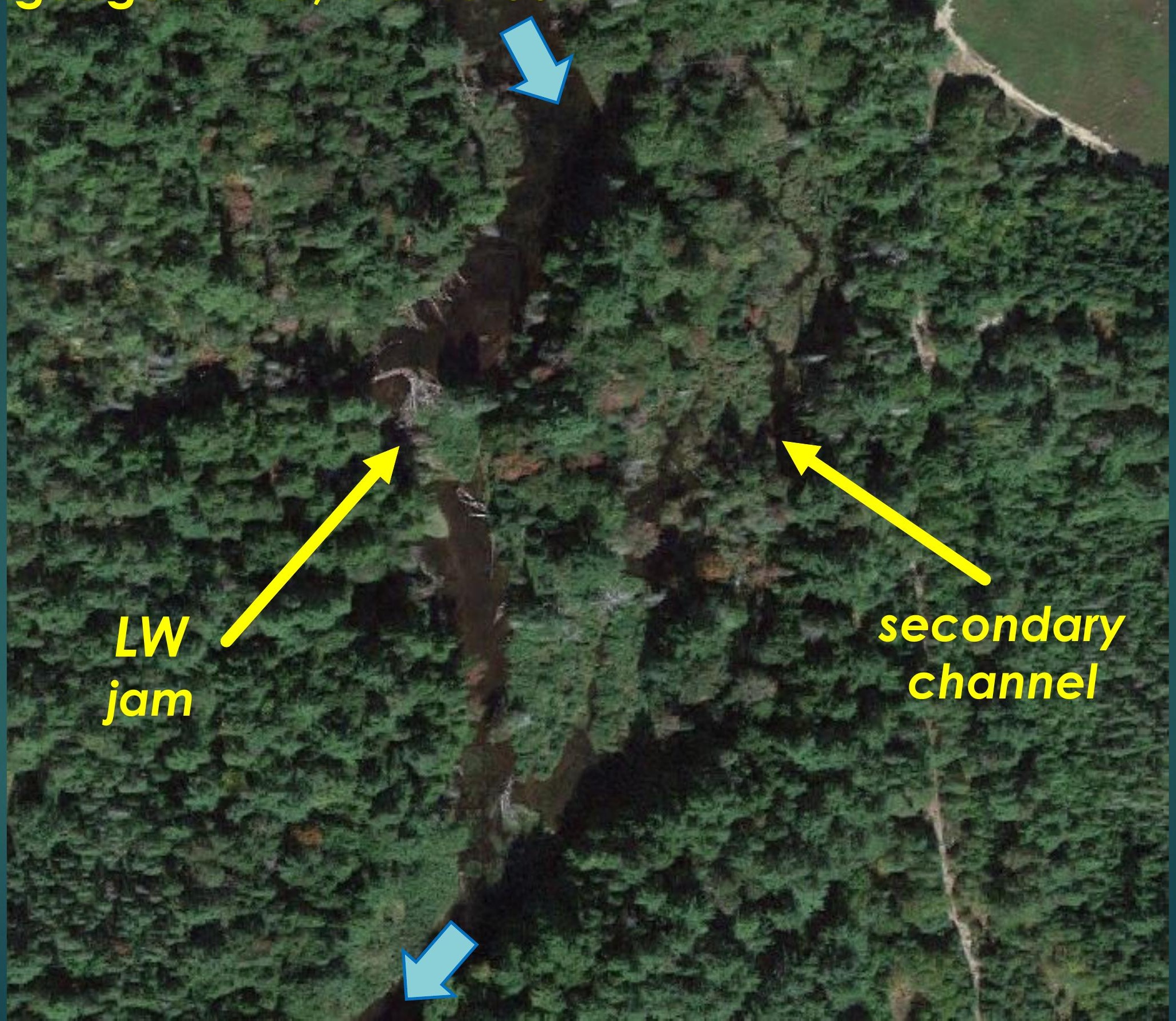




\section{BACl: Before, After Control, Impact}

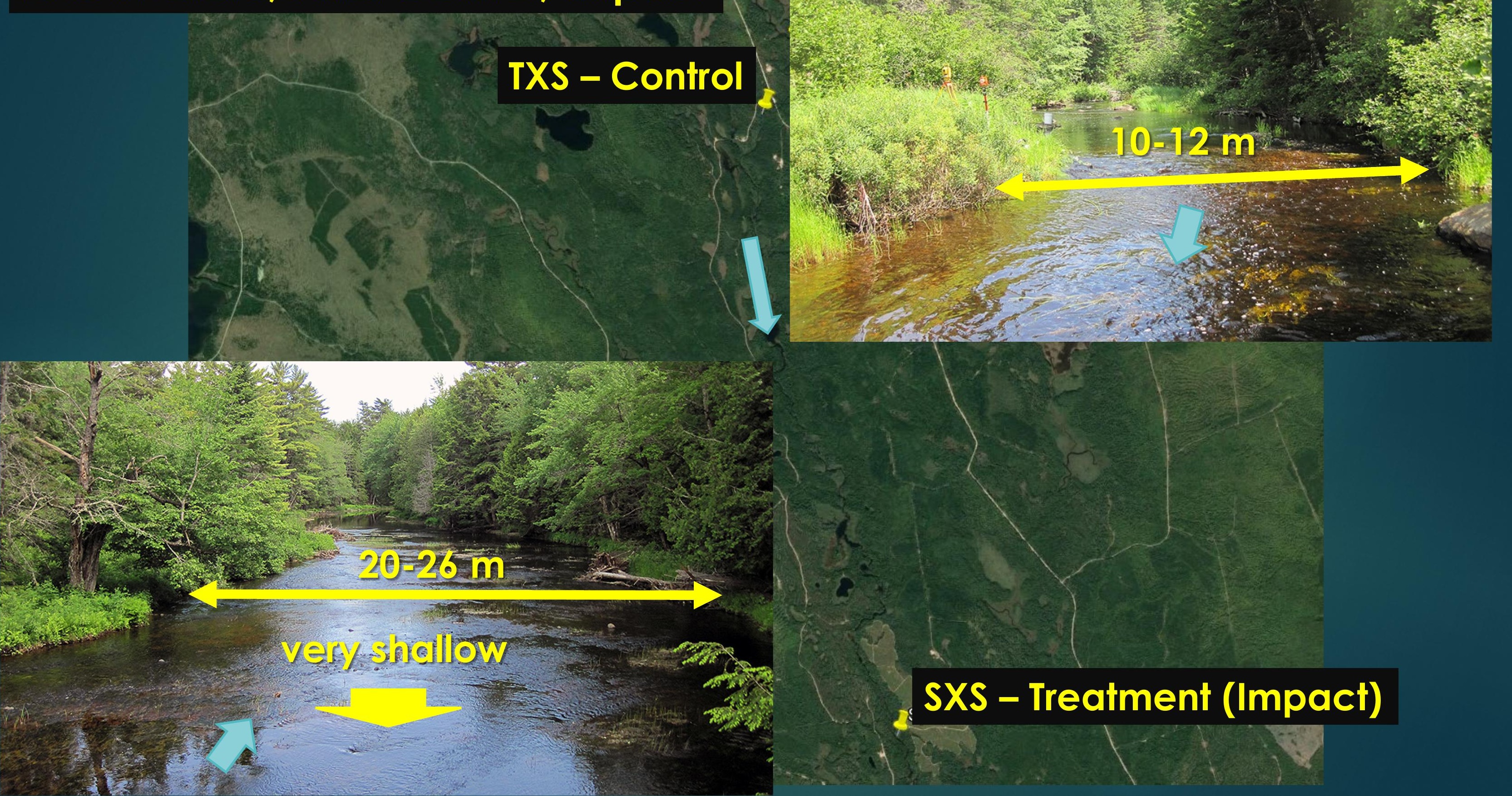




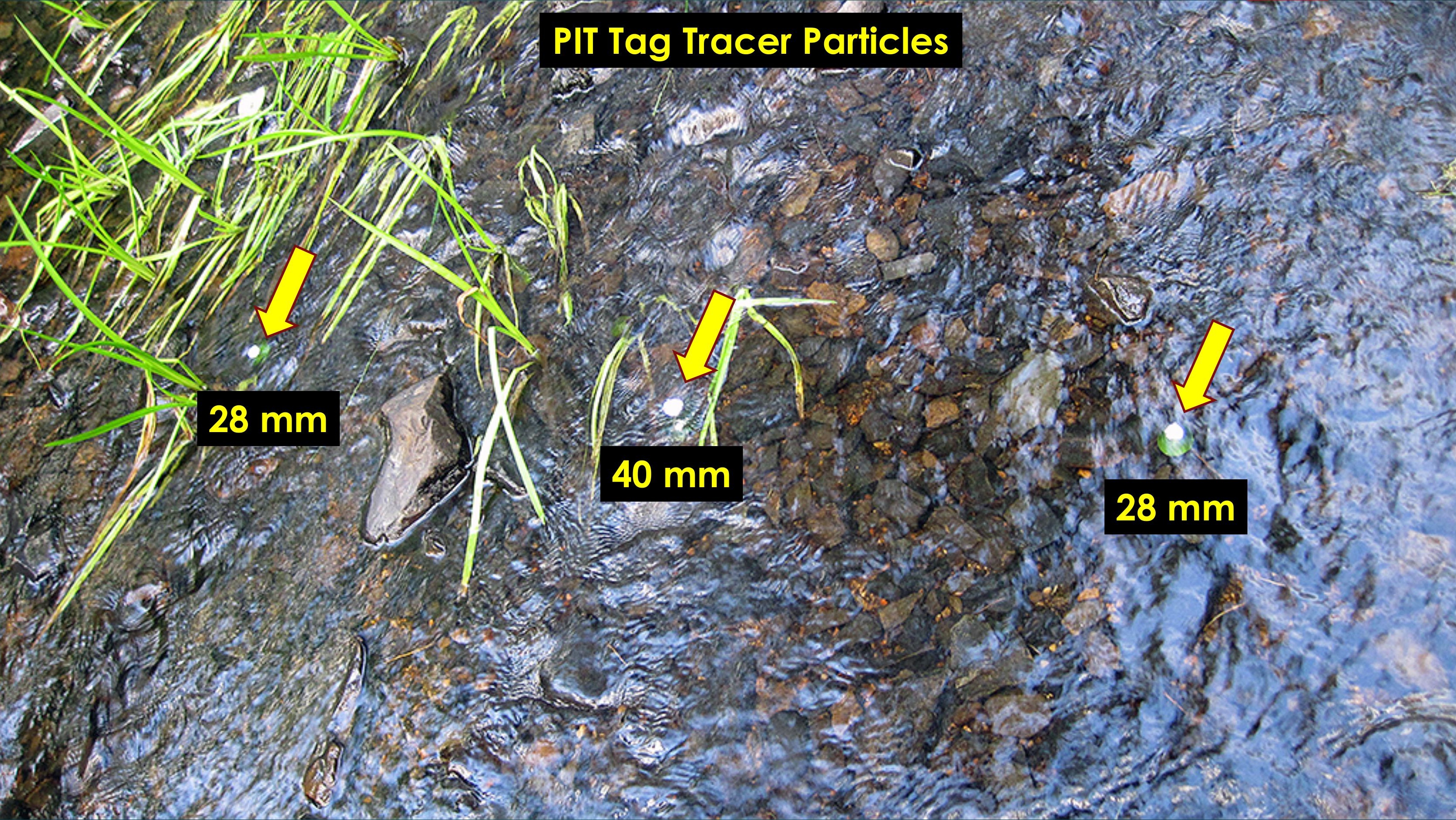




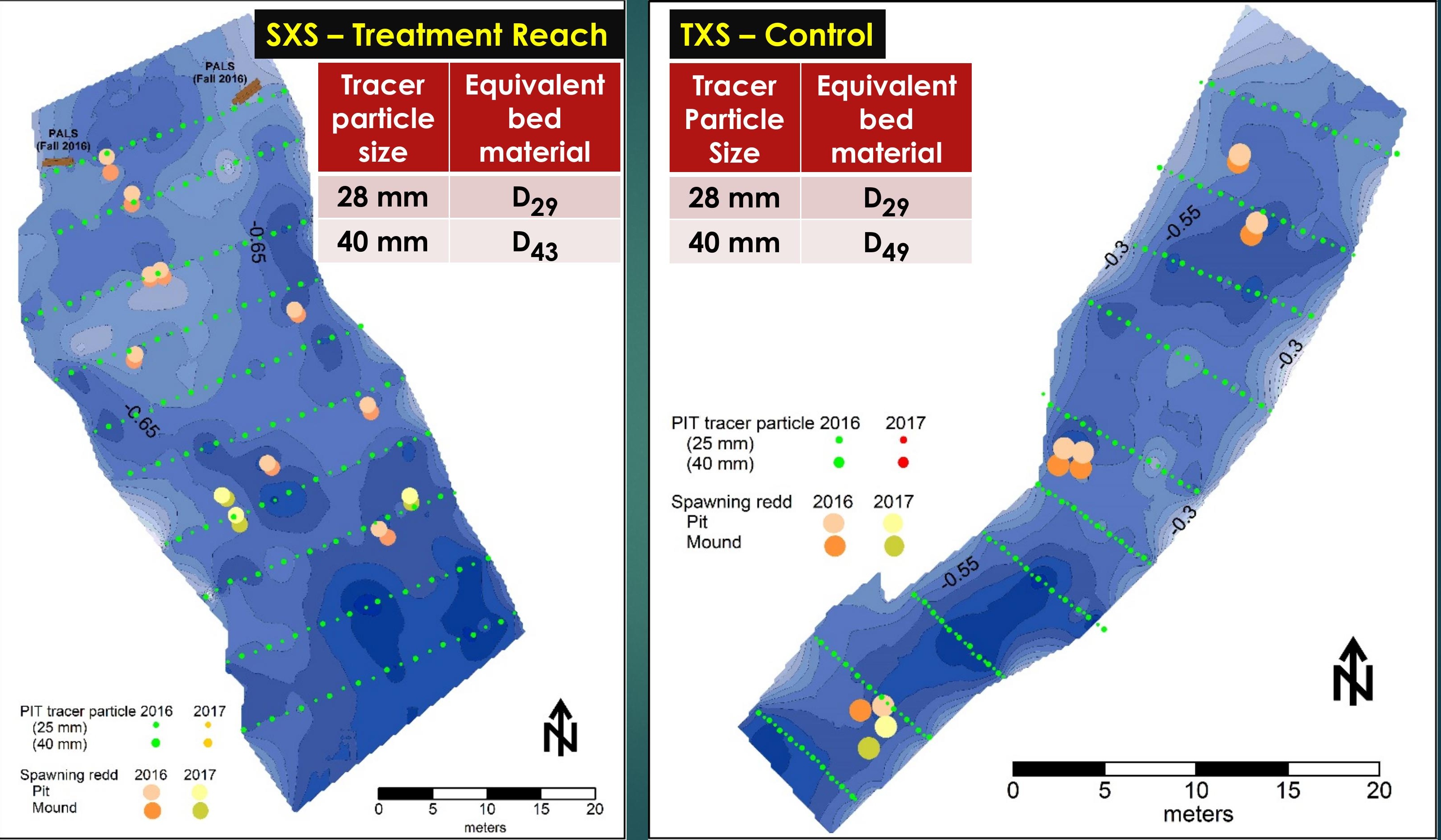




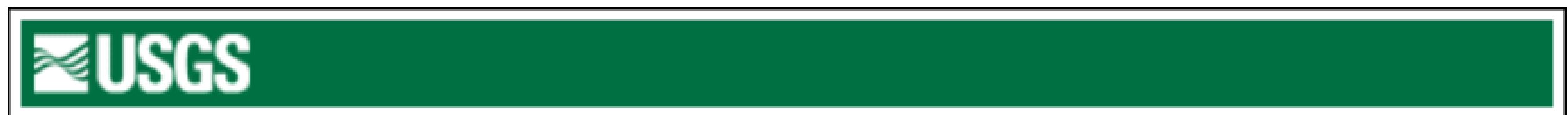

USGS 01022500 Narraguagus River at Cherryfield, Maine

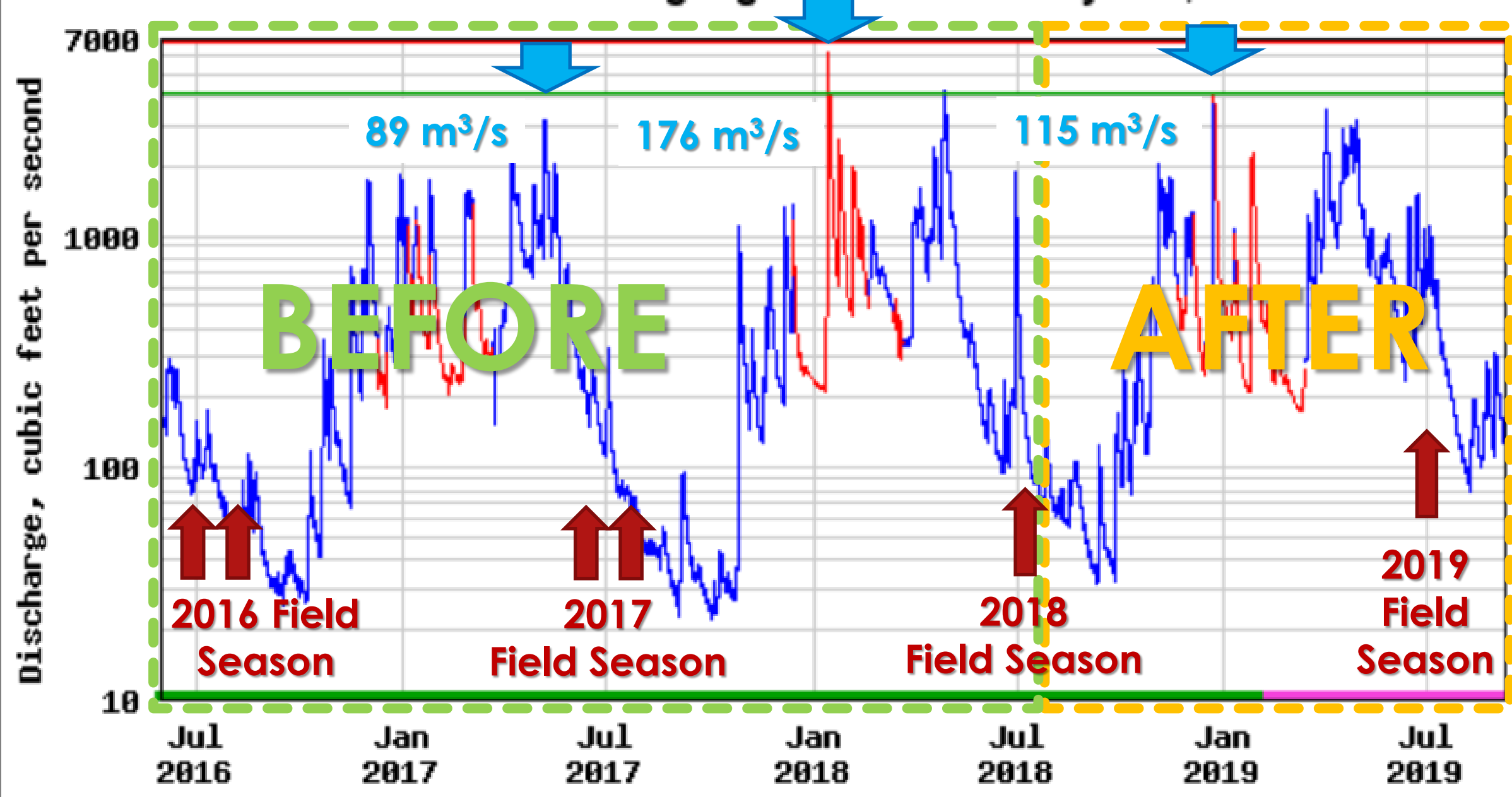

- Discharge

- Period of provisional data

- Estinated discharge

- 10 Year Peak Flow *

- Period of approved data

- 2 Year Peak Flow* 


\section{Cross-section resurveys}

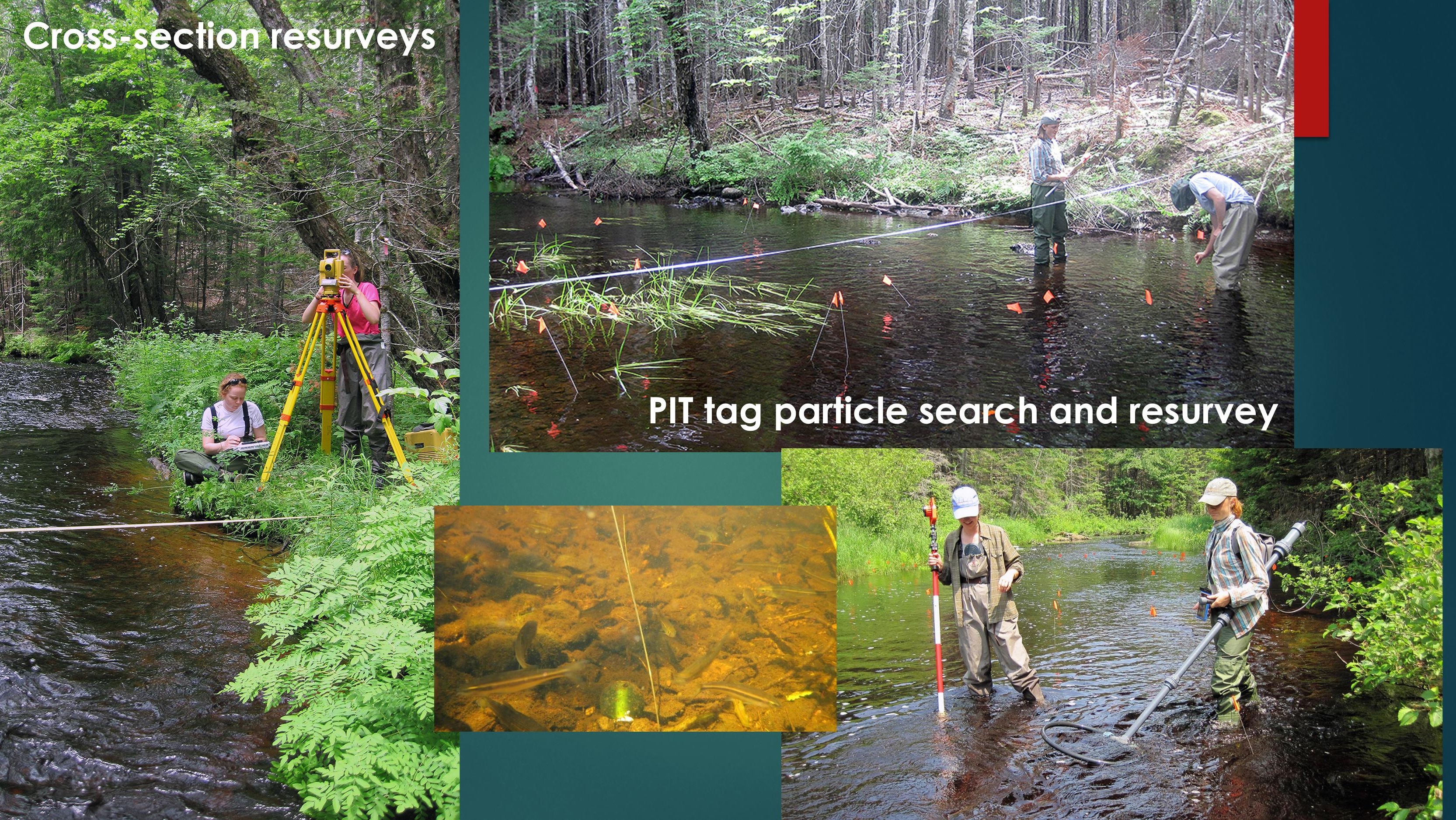




\section{Control \\ 2018 high water marks}
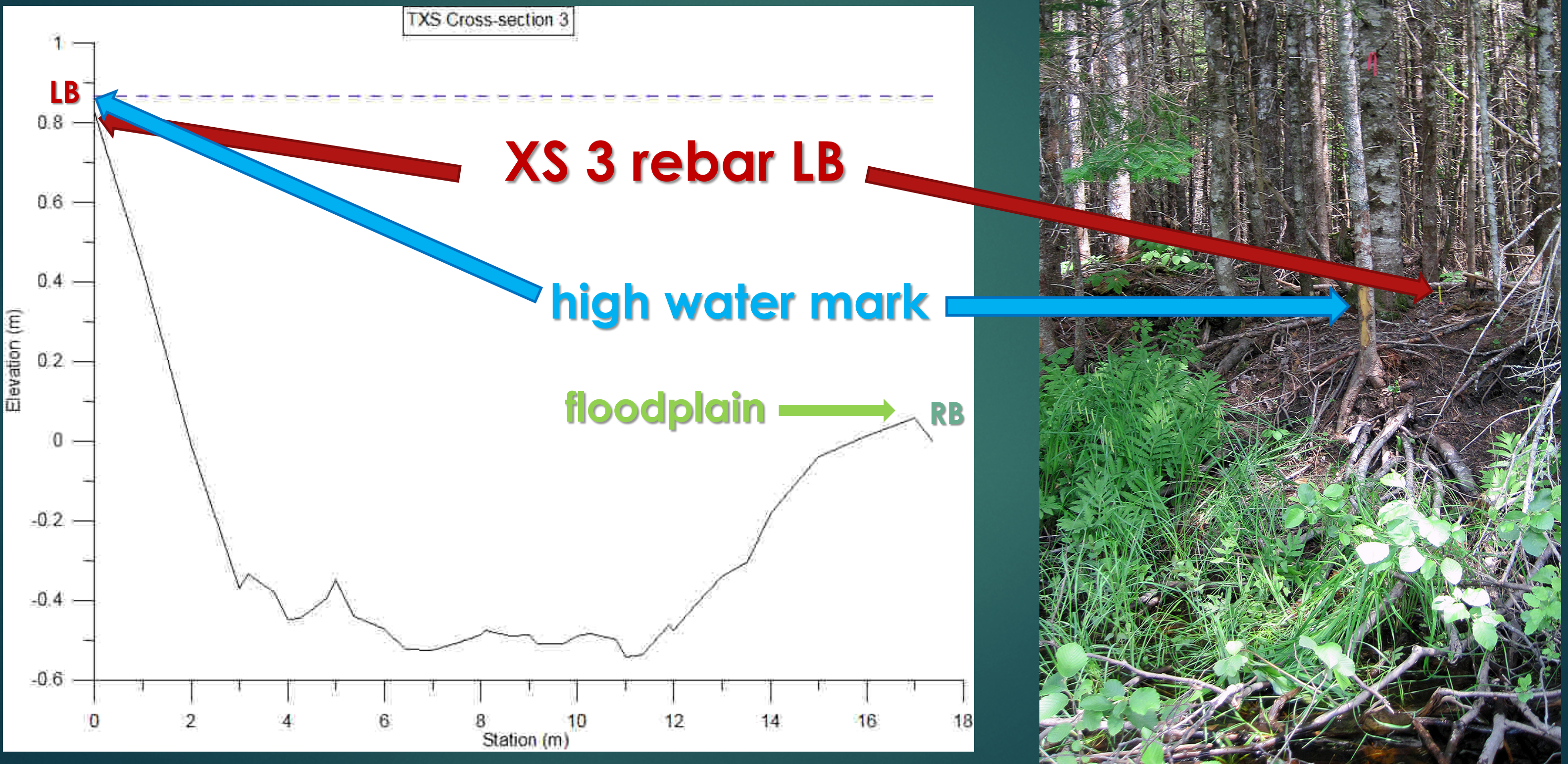


\section{Treatment (IMPACT): July 2018}

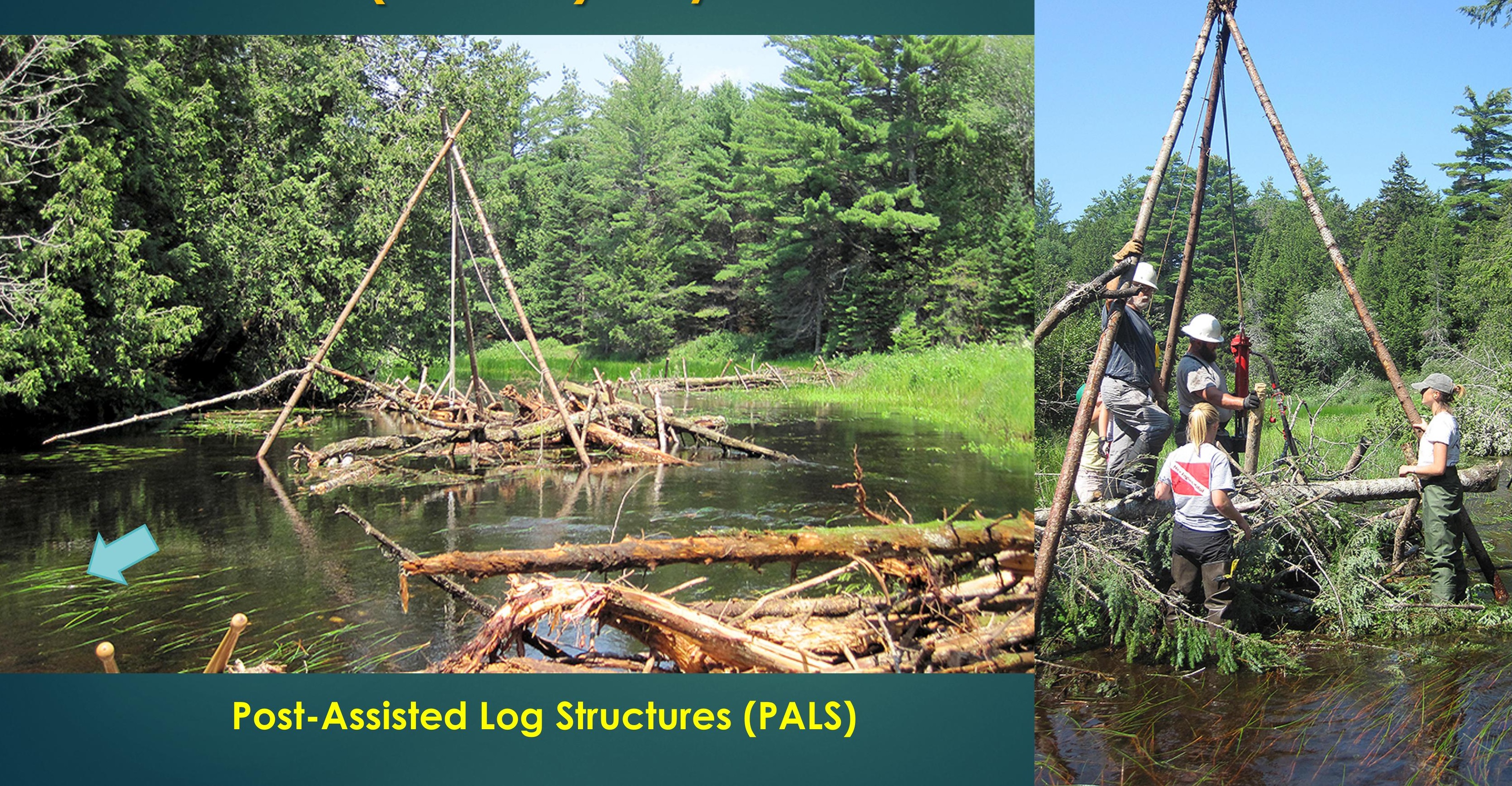




\section{Treatment (IMPACT): July 2018}

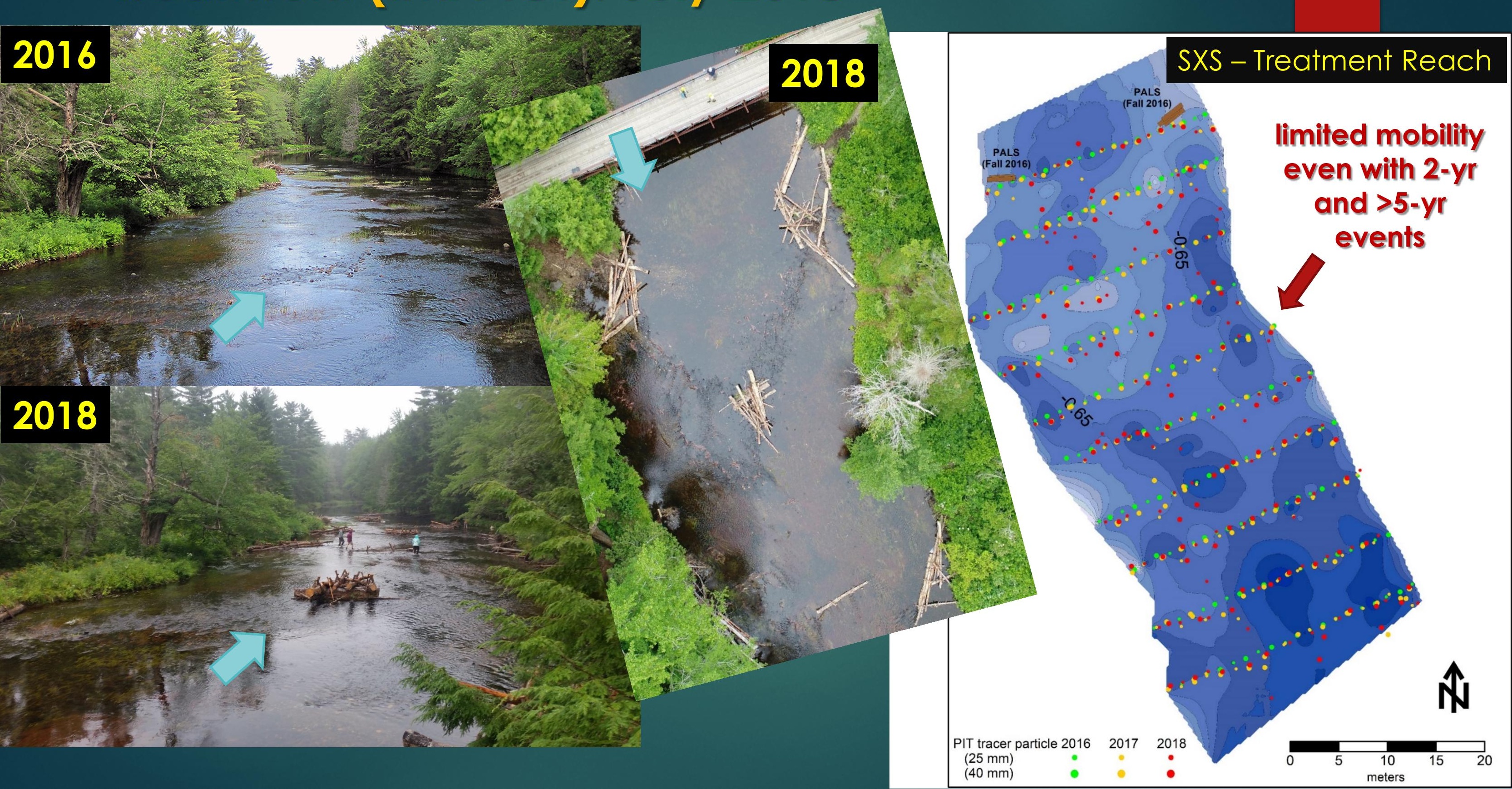


Treatment Reach

PIT tracer particle seeding locations

$$
20162018
$$

$28 \mathrm{~mm}$

$40 \mathrm{~mm}$

PIT tracer particle locations

$\begin{array}{llll}28 \mathrm{~mm} & 2017 & 2018 & 2019 \\ 40 \mathrm{~mm} & & \bullet & \bullet\end{array}$
Control Reach

\section{Bed elements}

Stable LW

LW moved 2018-2019

LW moved 2016-2018

Boulders

Redd pit and mound locations
Pit
2016
20172018
Mound

Recovery Rate (vs previous year)

\begin{tabular}{|c|c|c|}
\hline Year & $28-\mathrm{mm}$ & $40-\mathrm{mm}$ \\
\hline 2017 & $99(\mathrm{NA})$ & $99(\mathrm{NA})$ \\
\hline 2018 & $92(93)$ & $97(98)$ \\
\hline 2019 & $96(102)$ & $89(92)$ \\
\hline
\end{tabular}


Nominal logistic analysis

(Entrainment rates)

Reach $(p<0.001)$

Peak discharge $(p=0.0329)$

Particle size (Not Significant)

Treatment period (Not Significant)

Entrainment rate (re-entrained)

\begin{tabular}{|c|c|c|}
\hline Year & $28-\mathrm{mm}$ & $40-\mathrm{mm}$ \\
\hline 2017 & $13.1(\mathrm{NA})$ & $8.2(\mathrm{NA})$ \\
\hline 2018 & $17.4(4.3)$ & $22.9(0.0)$ \\
\hline 2019 & $13.2(2.5)$ & $10.2(0.8)$ \\
\hline
\end{tabular}

Entrainment rate (re-entrained)

\begin{tabular}{|c|c|c|}
\hline Year & $28-\mathrm{mm}$ & $40-\mathrm{mm}$ \\
\hline 2017 & $24.6(\mathrm{NA})$ & $29.4(\mathrm{NA})$ \\
\hline 2018 & $22.1(7.1)$ & $48.4(14.5)$ \\
\hline 2019 & $20.9(13.0)$ & $28.6(15.9)$ \\
\hline
\end{tabular}


PIT tracer particle locations

\section{Treatment Reach
The}

Change in embeddedness

2019-2018

100

80

60
40

40
20

0
-20

$-20$

-40
-60
-80
-100

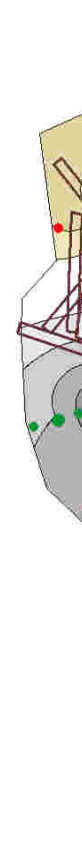

Stable

18-19

Moved

$28 \mathrm{~mm}$

$40 \mathrm{~mm}$

Redd pit and mound locations 2019

Pit

Mound

Bed elements

Stable LW

LW moved 2018-2019

LW moved 2016-2018

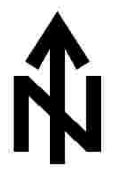

Bankfull depth estimate of particle entrainment size

$<28$-mm tracer 28-mm tracer 40-mm tracer sediment D50

$\square$
$\square$




\section{Tracer Particle Deposition}

\section{Treatment Reach}

Control Reach

PIT tracer particle depositional locations

$\begin{array}{lccc}28 \mathrm{~mm} & 2017 & 2018 & 2019 \\ 40 \mathrm{~mm} & \bullet & \bullet & \bullet\end{array}$

Redd pit and mound locations

\begin{tabular}{lllll} 
Pit & 2016 & 2017 & 2018 & 2019 \\
Mound & & & & \\
\hline
\end{tabular}

Bed elements

Stable LW

LW moved 2018-2019

LW moved 2016-2018

Boulders
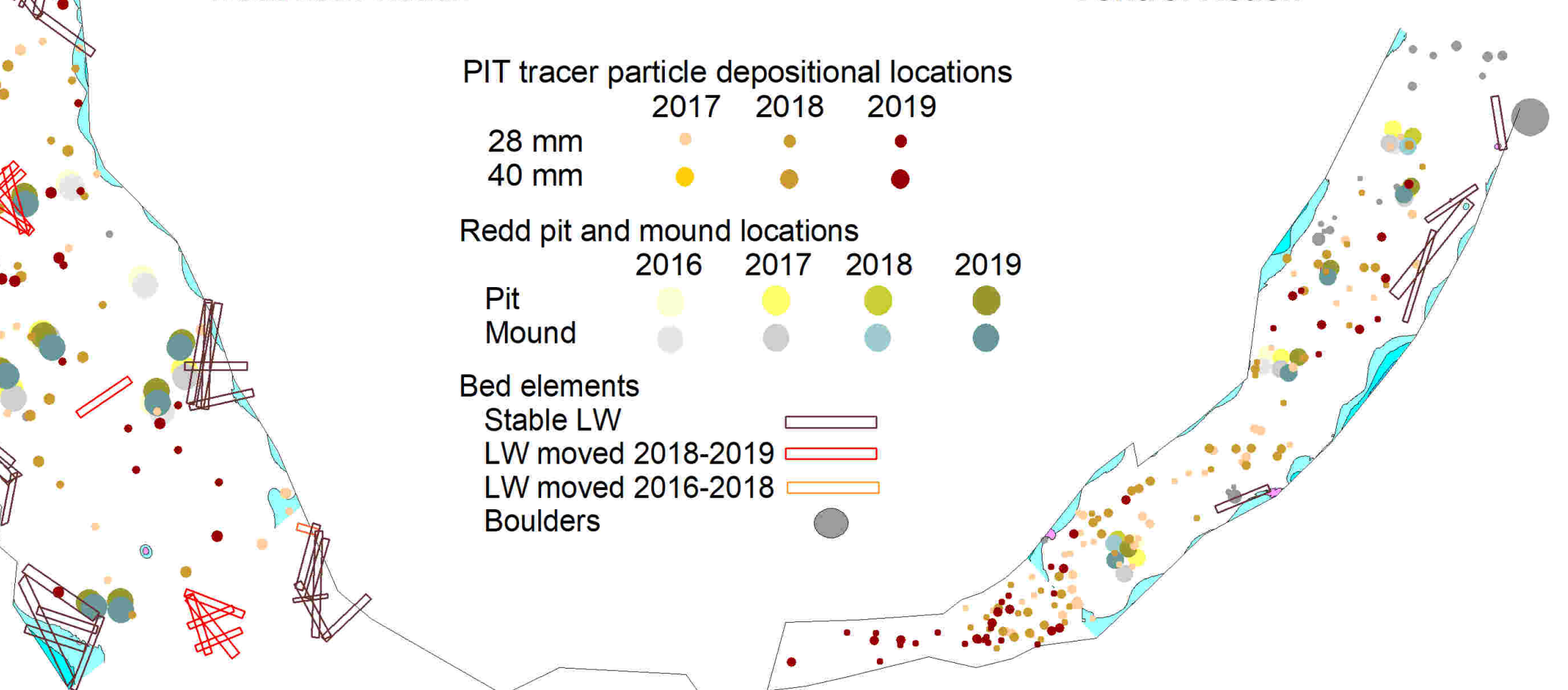

(
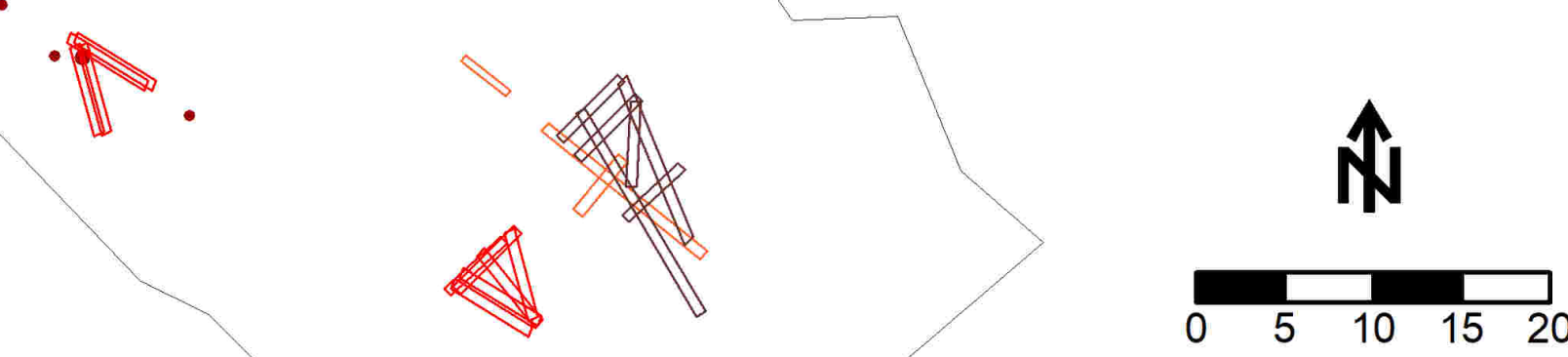

evation

change

$0.60 \mathrm{~m}$

$0.48 \mathrm{~m}$

$0.36 \mathrm{~m}$

$0.24 \mathrm{~m}$

$0.12 \mathrm{~m}$

$-0.12 \mathrm{~m}$

$-0.24 \mathrm{~m}$

$-0.36 \mathrm{~m}$

$-0.48 \mathrm{~m}$

$-0.60 \mathrm{~m}$ 


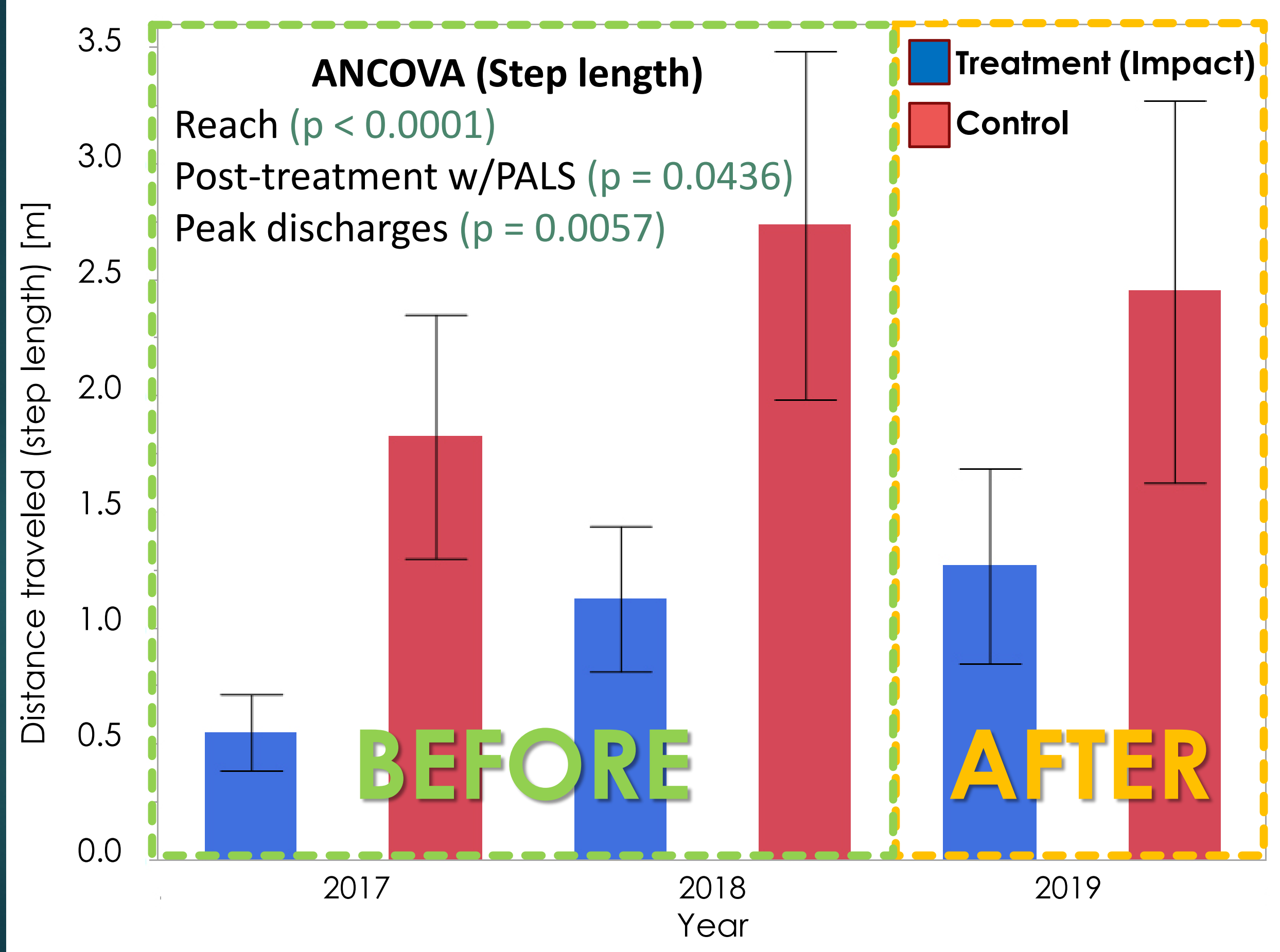




\section{Treatment Reach}

PIT tracer particle locations

2016-19 pre-2019

Stable Entrained Deposited Entrained Deposited

$28 \mathrm{~mm}$

$40 \mathrm{~mm}$

Redd pit and mound locations

$\begin{array}{lllll}\text { Pit } & 2016 & 2017 & 2018 & 2019 \\ \text { Mound } & & & & \\ & & & & \end{array}$

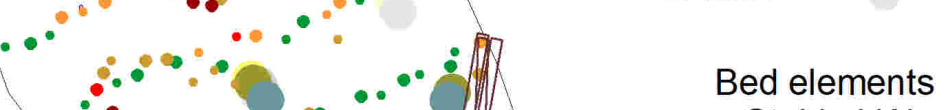

Stable LW

LW moved 2018-2019

LW moved 2016-2018

Boulders

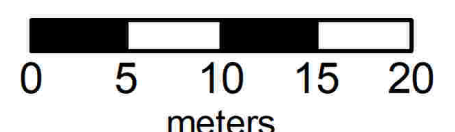

Elevation

change

$0.60 \mathrm{~m}$

$0.48 \mathrm{~m}$

$0.36 \mathrm{~m}$

$0.12 \mathrm{~m}$

$-0.12 \mathrm{~m}$

$-0.12 \mathrm{~m}$

$-0.24 \mathrm{~m}$

$-0.36 \mathrm{~m}$

$-0.48 \mathrm{~m}$

$-0.60 \mathrm{~m}$

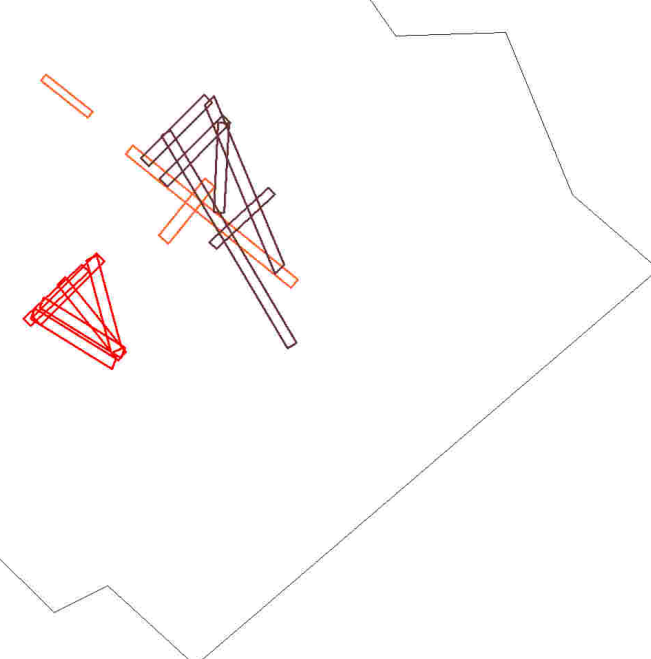


- The Narraguagus River is overly wide and shallow due to past logging activities

- Particle mobility is limited even during a large flood

- Particle entrainment rates between $\mathbf{2 8} \mathrm{mm}$ and $\mathbf{4 0}$ $\mathrm{mm}$ particles are similar (equal mobility?)

- PALS additions did NOT impact entrainment rates, but DID increase particle step lengths in the treatment reach 


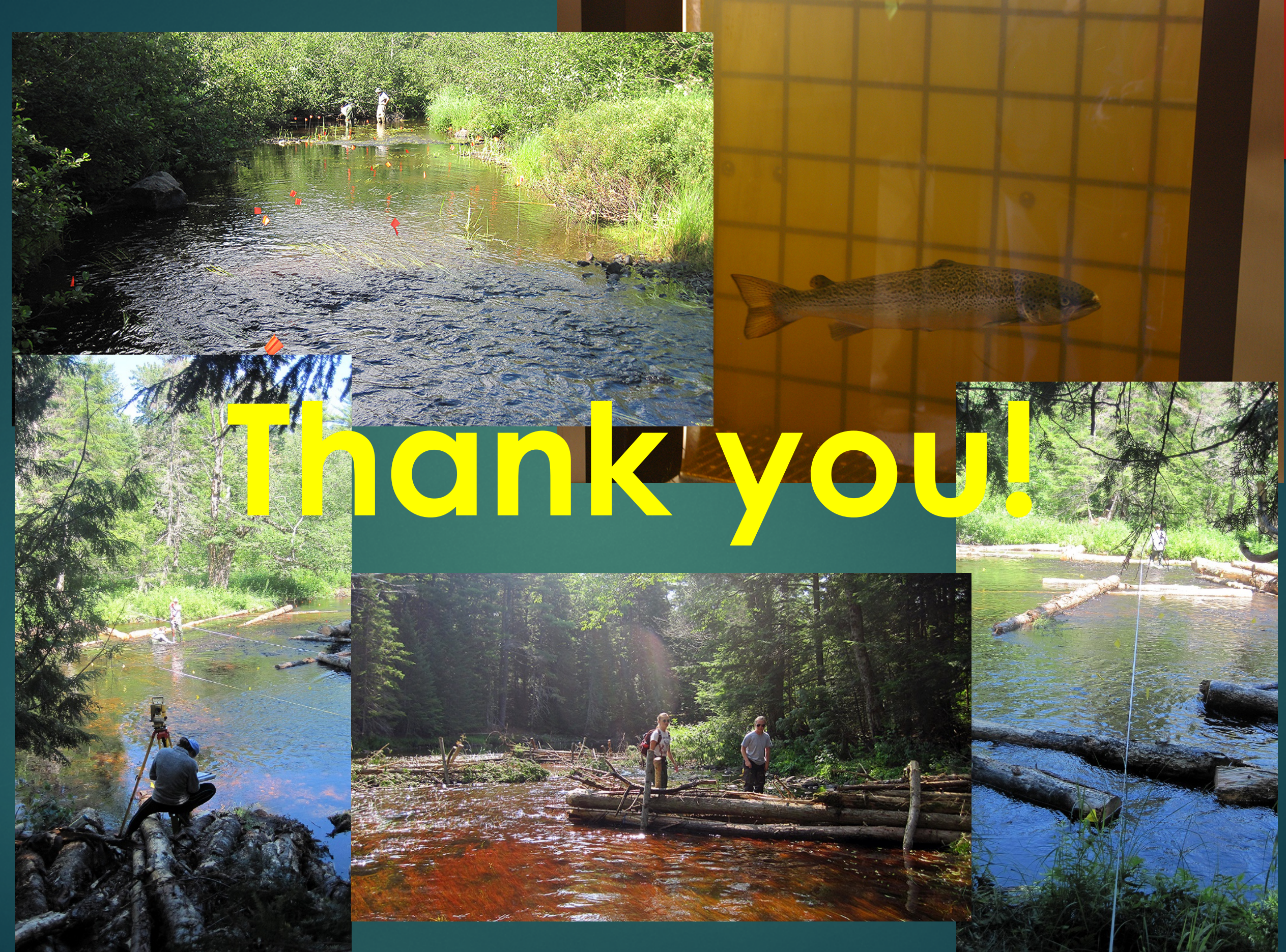




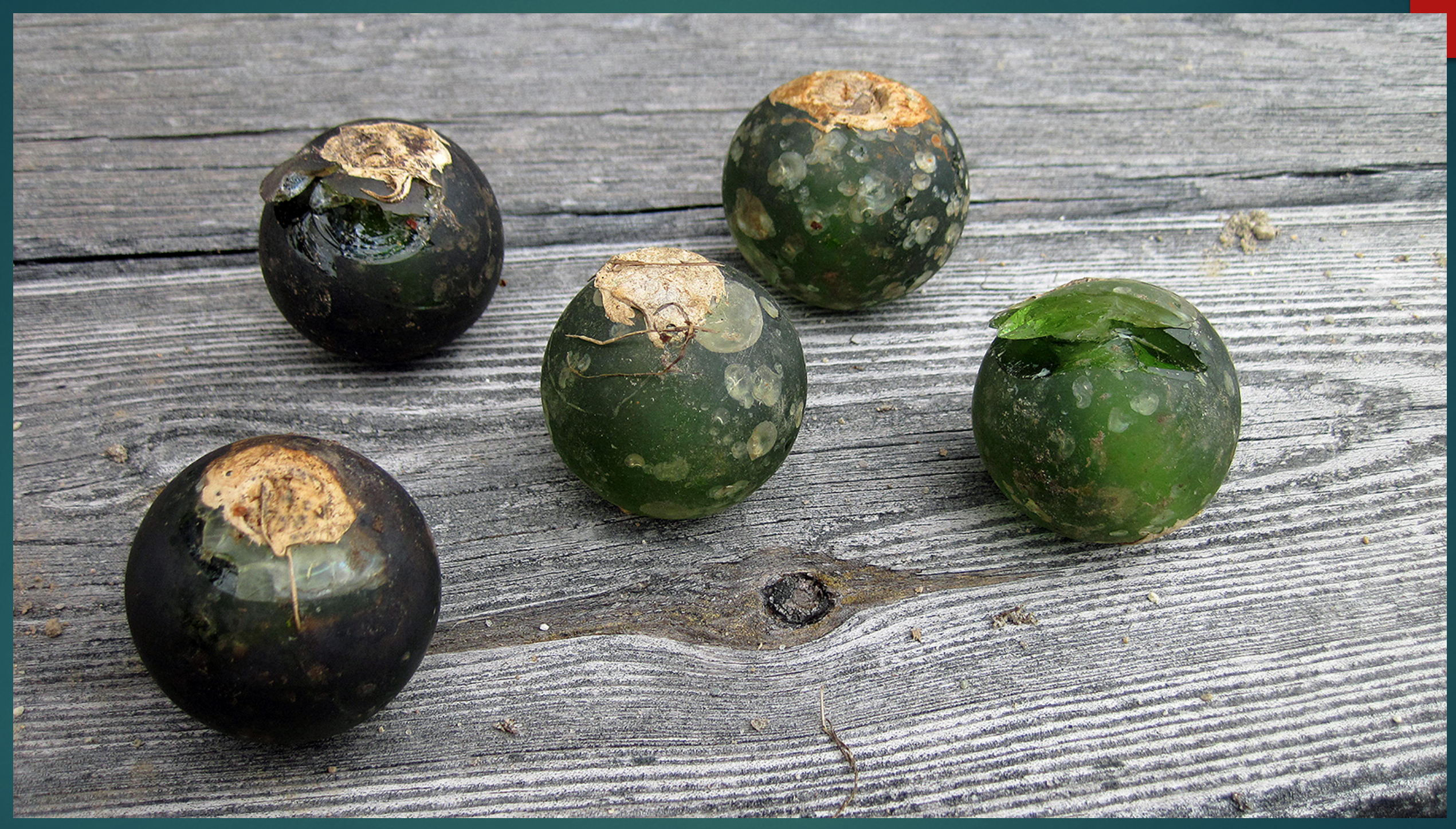




\section{Elevation Change: Treatment}

Elevation Change

2017-2016

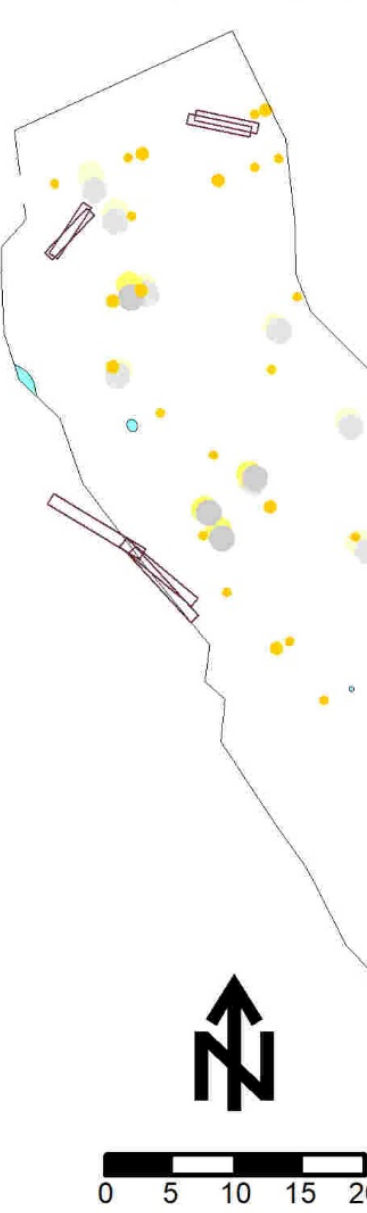

meters
Elevation Change

2018-2017

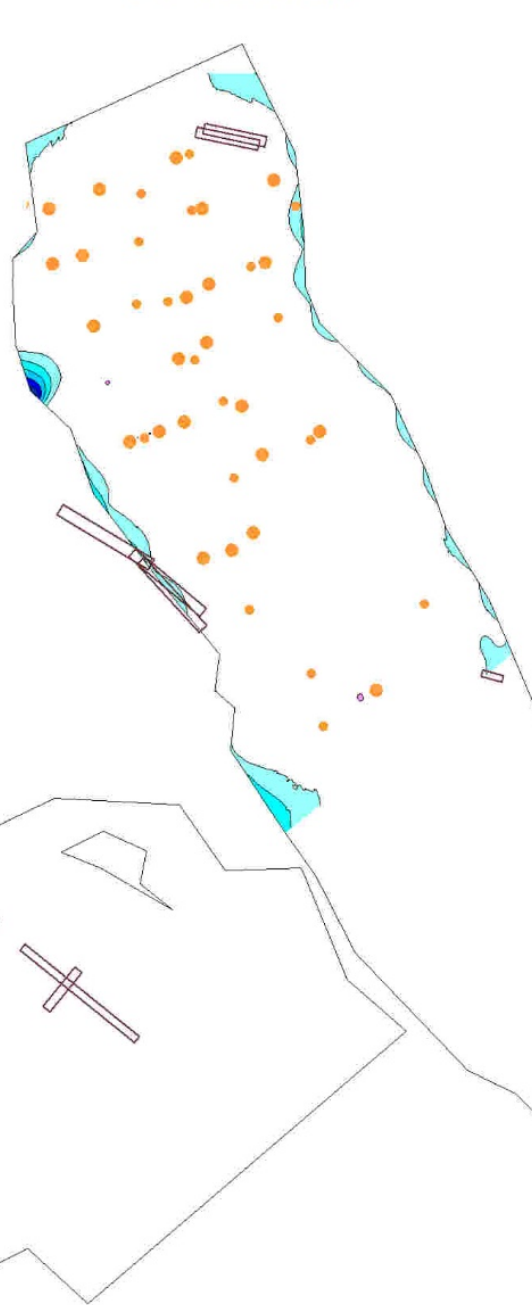

Elevation Change 2019-2018

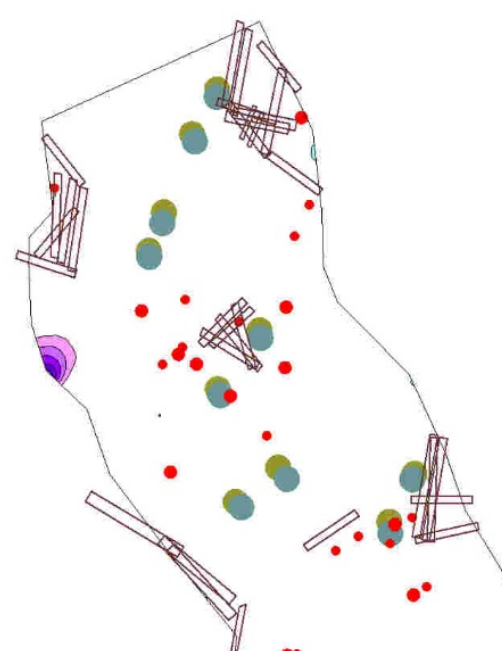

Elevation

change

$0.60 \mathrm{~m}$

$0.48 \mathrm{~m}$

$0.36 \mathrm{~m}$

$0.24 \mathrm{~m}$

$0.12 \mathrm{~m}$

$-0.12 \mathrm{~m}$

$-0.24 \mathrm{~m}$

$-0.48 \mathrm{~m}$

$-0.60 \mathrm{~m}$

PIT tracer particle entrainment locations

Redd pit and mound locations

$\begin{array}{lccclllll}28 \mathrm{~mm} & 2017 & 2018 & 2019 & & 2016 & 2017 & 2018 & 2019 \\ 40 \mathrm{~mm} & 0 & & \bullet & \text { Pit } & & & & \end{array}$

